\title{
Utility of multicolor fluorescent in situ hybridization in clinical cytogenetics
}

Syed M. Jalal, PhD and Mark E. Law, BS

\begin{abstract}
Purpose: Multicolor FISH (M-FISH) was introduced in 1996 to scan all 24 chromosomes in different fluorescent colors by use of a specific filter set and computer software. However, the clinical utility of M-FISH has been limited because of the lack of commercial availability of reagents and hardware. We have evaluated M-FISH for identification of markers, derivative chromosomes, and complex karyotypes. Methods: We present our findings based on a representative sample of one normal and six abnormal cases from a variety of tissue types. The results of M-FISH were confirmed by other well-established FISH probes. Results: M-FISH analyses were successful in all six cases. The derivative chromosomes, ring, and a complex karyotype were resolved. Conclusions: We find M-FISH to be an invaluable tool for a high degree of accuracy and efficiency for chromosome identification. The limitations similar to spectral karyotyping system (SKY) include the inability to detect intrachromosomal anomalies, abnormalities involving the p-arms of acrocentrics and areas rich in highly repetitive DNA. In addition, there are some concerns of misinterpretation due to overlap of fluorophore combinations of different chromosomes, especially for subtle insertional translocations. Genetics in Medicine, 1999;
\end{abstract} 1(5):181-186.

Key words: M-FISH, utility, clinical cytogenetics

Chromosome "painting" probes for visualization of entire specific chromosomes have been available since the late 1980 s. It is now possible to scan all of the chromosomes in defined colors simultaneously. By use of combinatorial labeling (Speicher et al. $\left.{ }^{1}\right)$ of five fluors $\left(2^{n}-1\right)$, it has become possible to assign specific fluor combinations to each human chromosome for its own spectral signature. Speicher et al. ${ }^{1}$ used five fluorophores, a series of high contrast fluorochrome specific filters, and computer software for simultaneous detection of all 24 human chromosomes in unique colors. Eils et al. ${ }^{2}$ have proposed strategies to optimize labeling of the probes, filters, and image analysis. This technique has been variously called multicolor FISH, multifluor FISH, or multiplex FISH (M-FISH). We have opted to use the term multicolor FISH. Schrock et al. ${ }^{3}$ introduced the scanning of entire human genome at defined spectral emission for each chromosome, to which they referred as multicolor spectral karyotyping or SKY. SKY also uses combinatorial labeling, but instead of filters it uses Fourier spectroscopy and chargedcoupled-device (CCD) imaging to measure emission spectra. The spectral signature of each chromosome is translated to vivid colors by the help of computer software.

\footnotetext{
Fran she Cytogenetics Laboratory. Departmon of Luburatory Medicine and Pathologe: Mayo Climic and Mayo Foundation, Reschester, Minmesoled

Any reference to products in this report docs not imply endorsement of the Mayo Formetition.

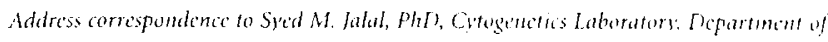

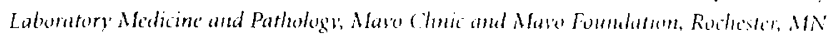
55905 .

Recived: March 22, 1999

Accopted: May. 2s, 1999
}

M-FISH software has become commercially available for incorporation in the existing image capture systems for FISH. We present our experience with M-FISH for its utility in routine clinical cytogenetics. We believe that such a system (SKY or M-FISH) is invaluable for analysis of derivative chromosomes, markers, and complex karyotypes.

\section{METHODS}

A variety of tissue sources were used for M-FISH analysis including fibroblasts from products of conception, amniocytes (AF), peripheral blood, and bone marrow. The samples were cultured and harvested using standard cytogenetic procedures. Slides were prepared by dropping a single drop of cell suspension on a dry microscope slide in a Thermatron (Holland, MI) drying chamber ${ }^{4}$ with defined temperature and relative humidity for each tissue type. The slides were placed in $0.005 \%$ pepsin $/ 0.01 \mathrm{NHCL}$ at $37^{\circ} \mathrm{C}$ for $0-2$ minutes, jet air dried, artificially aged in a $90^{\circ} \mathrm{C}$ drying oven for 5-10 minutes, placed in $2 \times \mathrm{SSC}$ at $37^{\circ} \mathrm{C}$ for $15-60$ minutes, and dehydrated through ethanol series $(70 \%, 85 \%, 100 \%)$ at room temperature for 1 minute in each solution and then jet air dried. The M-FISH reagent was placed on the slide, coverslipped, sealed with rubber cement, and placed in a humidified HYBrite (Vysis, Downers Grove, IL) with the following settings: melting temperature of $80^{\circ} \mathrm{C}$, melting time of 0.03 minutes, hybridization temperature of $37^{\circ} \mathrm{C}$, and hybridization time of 20 hours. It is especially important that the slides are denatured and pretreated properly. If they are under denatured or aged too long, the hybridization will be insufficient, and if they are over denatured or not artificially aged enough, chromosome morphology will be compromised. If there is too much cytoplasm left, 
there will be too much background, especially from the CY5 fluor filter.

If the slides were G-banded, immersion oil (we do not use a coverslip) was removed with xylene, and the slides were destained using 3:1 methanol to acetic acid fixative for 10-15 seconds and jet air dried. The slide was placed in $2 \times \mathrm{SSC}$ for $5-15$ minutes at $37^{\circ} \mathrm{C}, 1 \%$ formaldehyde ( $1 \%$ formaldehyde/ phosphate balanced saline, $\mathrm{pH}$ 7.4) for 5 minutes at room temperature, balanced phosphate buffer (PBS) for $5 \mathrm{~min}$ utes at room temperature, and treated in room temperature ethanol dehydration series $(70 \%, 85 \%, 100 \%)$ for 1 minute in each solution. M-FISH probe $(10 \mu \mathrm{L})$ was placed on the hybridization site, coverslipped, sealed with rubber cement, and placed in an HYBrite with the same settings used for fresh slides ( see above). With both methods, the hybridization was allowed to proceed overnight in a HYBrite or humidified chamber at $37^{\circ} \mathrm{C}$. The G-banding method includes artificially aging the slides for 1 hour at $90^{\circ} \mathrm{C}$, trypsinizing, and staining with Leishman's stain.

After hybridization, the slides were washed in $0.4 \times \mathrm{SSC}$ at $73^{\circ} \mathrm{C}$ for 2 minutes, rinsed in $2 \times \mathrm{SSC} / 0.1 \% \mathrm{NP} 40$ at room temperature for 5-30 seconds, jet air dried, and counterstained with DAPI ( $100 \mathrm{ng}$ DAPI $/ \mathrm{mL}$ of antifade). The slides were viewed with a Zeiss microscope powered by a $100-\mathrm{W}$ mercury bulb. Vysis (Downers Grove, IL) M-FISH filter sets and software were used for capturing, viewing, and evaluation.

Slides were scanned with a $40 \times$ objective lens using the spectrum gold filter. When a suitable metaphase was identified, the image was captured by $100 \times$ objective. When the capture software is initiated, the user is asked to move the filter to the correct setting for the first fluorophore. After the first exposure is taken, the filter is moved again for the next fluorophore and so on until all five fluorophores and the DAPI counterstain have been individually exposed. The software then merges these images into one image. The chromosomes are classified according to the fluorescence pattern detected by each filter. The manufacturer provides the information on the fluorophore signature for M-FISH classification based on fluorophore combination(s) of each chromosome. At least five metaphases were analyzed by M-FISH and the results were identical in all five for each of the six reported cases.

\section{RESULTS}

Standard cytogenetic analysis, M-FISH results, and further confirmation of the anomalies by whole chromosome painting probes and locus specific probes are presented in a representative series of cases from a variety of tissue types.

\section{Case 1}

Based on the banded chromosome analysis from PHA-stimulated blood, Case 1 was a normal male. M-FISH analysis depicts a normal karyotype, and every chromosome is displayed in its unique color. The chromosome edges, overlaps, and occasional background noise causes some blotching in the display colors (Figure 1a).

\section{Case 2}

A 27-year-old male was referred to rule out Klinefelter syndrome. The mother, who had a history of four multiple miscarriages, was chromosomally normal. Thirty GTG-banded metaphases were analyzed and two karyotyped as $46, \mathrm{X}$, add $(\mathrm{X})(\mathrm{p} 22.3)$. By M-FISH it was demonstrated that the abnormal segment in the $\operatorname{der}(\mathrm{X})$ was translocated from Y (Fig. lb). The translocation was confirmed by use of SRY probe.

\section{Case 3}

An amniotic fluid specimen was received due to intrauterine growth retardation for direct aneusomy FISH and standard chromosome analysis. The FISH analysis for numeric anomalies for chromosomes 13, 18, 21, $\mathrm{X}$ and $\mathrm{Y}$ from an

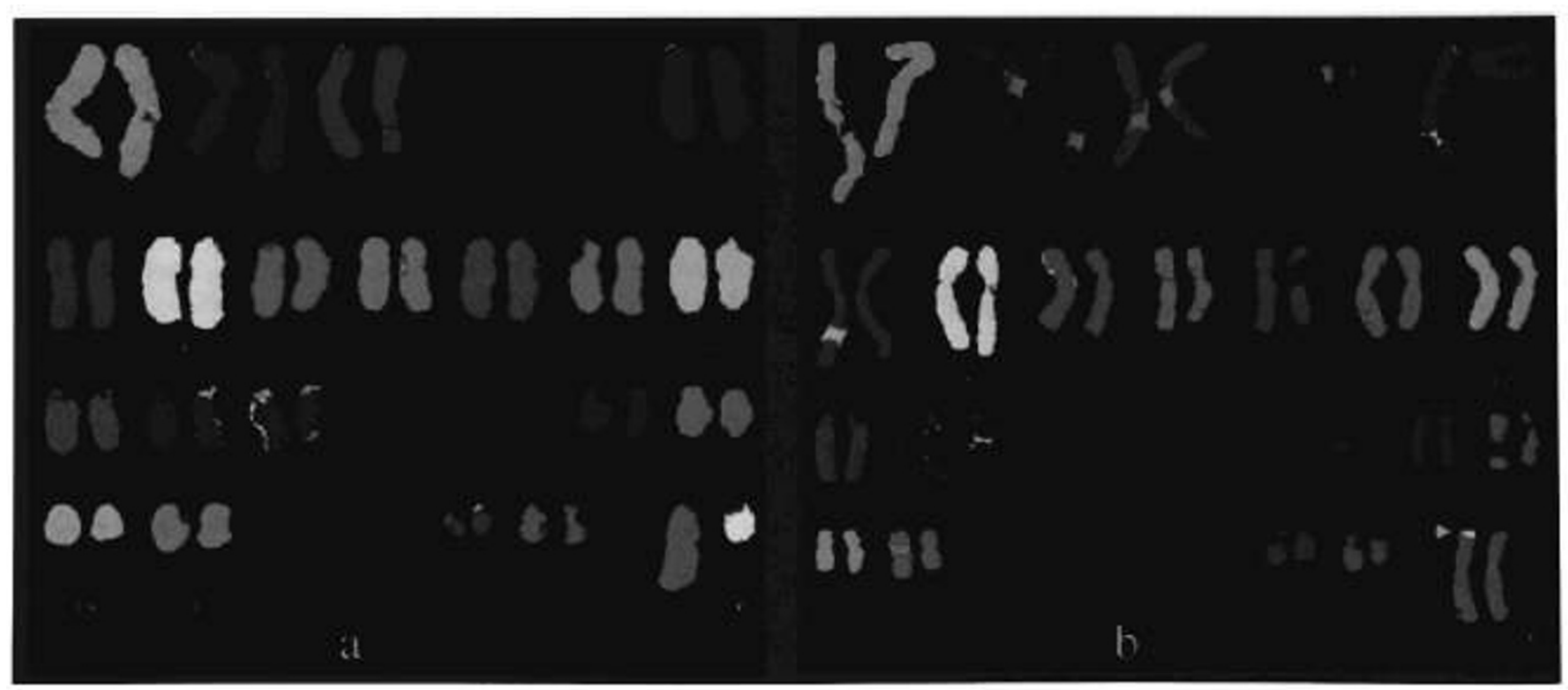

Fig. 1 M-FISH analysis from PHA-stimulated whole blood cultures. a, Normal male; b, 46, XX male. Translocation of a segment of Y chromosome to Xp22.3 was detected (arrow). 
uncultured AF sample had a normal autosomal pattern for a female. The chromosome analysis from 20 GTG-banded metaphases from five colonies (a slow grower) and four primary cultures was 46, XX, add (15) (q26.1). M-FISH indicated the translocated segment of der (15) to be from chromosome 9 . This was confirmed on the same metaphase by use of wcp 9 and wcp 15 (Figure 2, a and b). Use of the pl6 (9p21) probe further indicated the translocated segment of der (15) to be from the p-arm of chromosome 9. Both parents were karyotypically normal.

\section{Case 4}

An amniotic fluid specimen was received for chromosome analysis due to maternal age. Twenty GTG-banded metaphases from 15 colonies and three primary cultures were analyzed. The karyotype was $47, \mathrm{XX},+\mathrm{r}$. The ring was identified to be from chromosome 19 by M-FISH and confirmed by use of wcp 19 (Figure 2, $\mathrm{c}$ and d). Both parents were chromosomally normal.

\section{Case 5}

A 25-year-old miscarried at 7.5 weeks gestation (estimated by LMP). The fibroblast culture from the POC was analyzed. Twenty GTG-banded chromosomes revealed a karyotype of $46, X Y$, add (4) (p12). Analysis of sequential GTG followed by $\mathrm{M}$-FISH indicated the abnormal segment of chromosome 4 to be from chromosome 2 (Figure 3 , a and $b$ ). This was confirmed by use of wcp 2 . The mother was karyotypically normal and the father was unavailable for chromosome analysis.

\section{Case 6}

A bone marrow specimen from an 85-year-old female was received for chromosome analysis due to pancytopenia. Ten

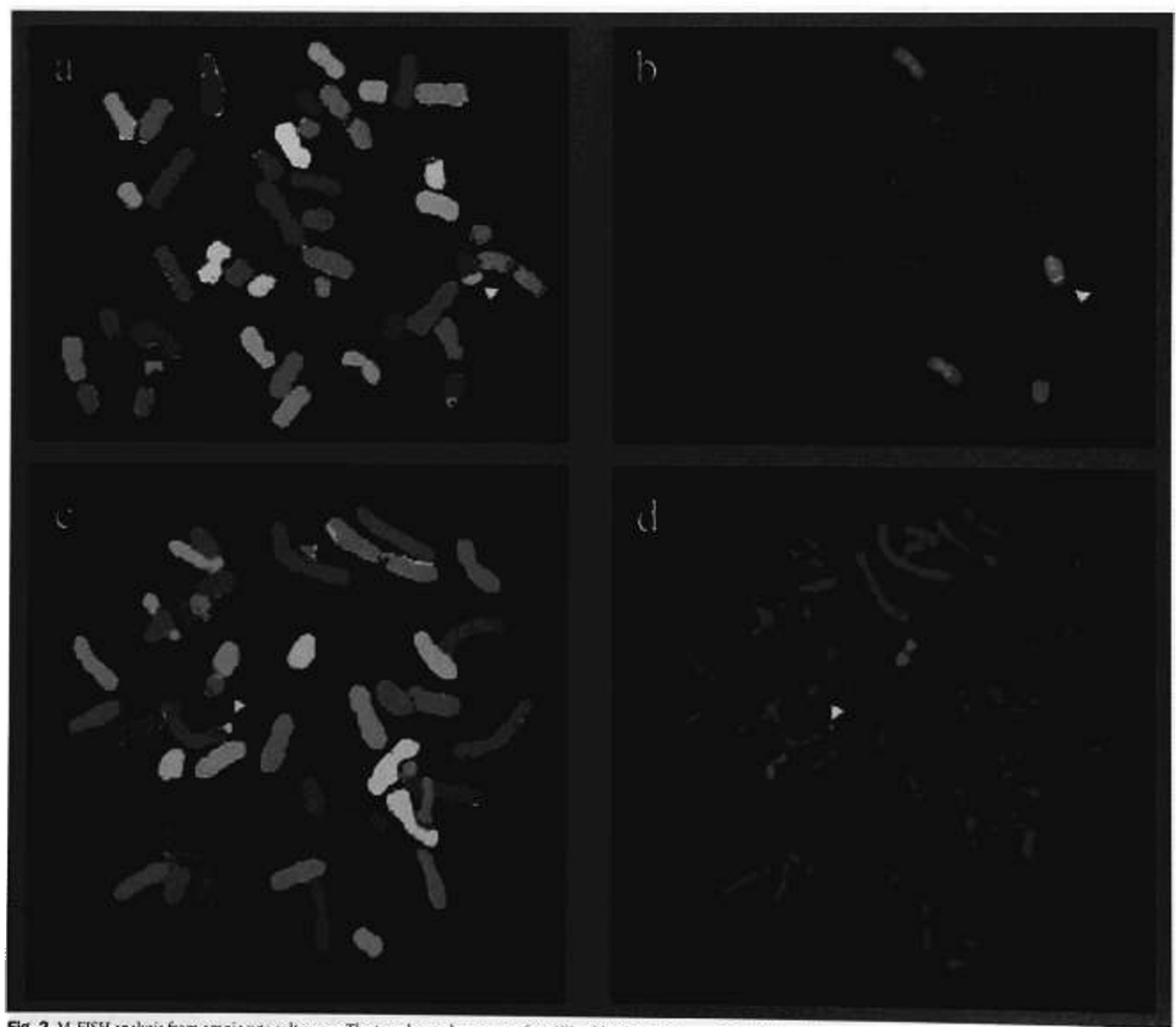

Fig, 2 M-FISH analysis from amaicyte cultures. a, The translocated segment of $46, X X$, add ( 15 i (q 26.1 i was identified to be from chromosome 9 (arrow). b, This finding was confirmed by the use of wcp 9 and wcp 15 (arrow). c, The ring from $47, \mathrm{XX}, \mathrm{r}$ was found to be from chromosome 19 (arrow). d, This was confirmed by use of wcpl 9 ( $a$ arrow). 

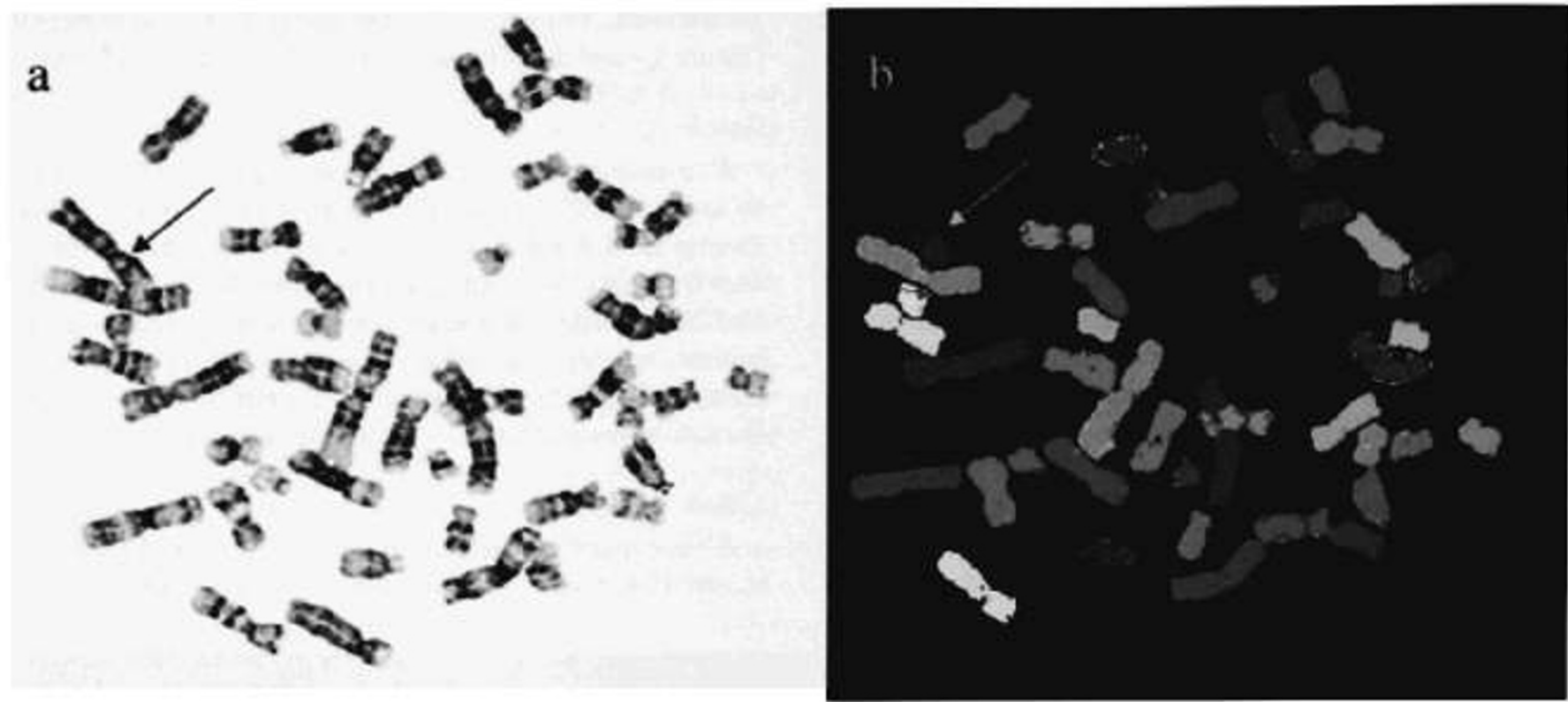

Fig. 3 Sequential N-FISH of previously GTG banded metaphase spread from fibroblast culture of a product of conception. a, GTG banded metaphase of 46, XY, add(4)(p12) (arrow). b. The same metaphase spread was analyzed by M-FISH and the translocated segment of der(4) was identified to be from chromosome 2 (arrow).

GTG- and 10 QFQ-banded metaphases were analyzed. The karyotype was $44-46, \mathrm{XX}$, add (2) (q37), -4,-5, add (9) (pl1), add (17) (p11.2), add(19) (p13.1),-20,-22,+24 mar [cp 19]/46, XX (1) consistent with the diagnosis of a neoplastic hematologic disorder. M-FISH analysis resolved all of the markers and derivative chromosomes (Figure 4): the add (2) was a complex translocation product of $\mathrm{t}(2 ; 4,9)$ two markers were identified as der (4) $t(4 ; 22)$, add (9) was $t(5 ; 9)$, add (17) was $\mathrm{t}(5 ; 17)$, add(19) was $\mathrm{t}(19 ; 20)$, and another marker was $\mathrm{t}(19 ; 20)$, which was not identified by QFQ or GTG banded analysis. Thus, the M-FISH based karyotype was $45, \mathrm{XX}$, der (2) $\mathrm{t}(2 ; 4 ; 9), \operatorname{der}(4) \mathrm{t}(4 ; 22) \times 2, \operatorname{der}(9) \mathrm{t}(5 ; 9), \operatorname{der}(17) \mathrm{t}(5 ; 17)$, $\operatorname{der}(19) t(19 ; 20), \operatorname{der}(20)(19 ; 20),-22$.

\section{DISCUSSION}

One of the more labor and time intensive efforts in clinical cytogenetics is to identify markers, derivative chromosomes, and analysis of complex karyotypes, both for congenital and hematologic disorders. It has been estimated that de novo structural rearrangements occur with a frequency of $0.70 / 1000$ rising to $2.4 / 1000$ among mentally retarded individuals. ${ }^{5}$ The prenatal frequency of de novo supernumerary markers (that includes bisatellited marker 15 ) is placed at $1 / 2500 .^{6}$ In order to assess their clinical significance, it is important to determine the origin of markers or derivative chromosomes. Recent efforts for such an identification has succeeded for a series of markers identified prenatally or postnatally when analysis of banded chromosome was combined with FISH probes specific for centromeres or specific chromosomes. ${ }^{7-12}$

With the advent of SKY, markers, derivative chromosomes, and complex karyotypes have been analyzed with relative ease. Reid et al., ${ }^{13}$ in an excellent review article on SKY, cite six papers on clinical cytogenetics and three on cancer cytogenetics among others. It is indeed exciting to be able to scan the entire genome (with the exception of acrocentric short arms and segments of highly repetitive DNA) by this technique. The limitation is, of course, the inability to detect intrachromosomal inversion, insertion, or subtle deletions. Use of conventional banding techniques, therefore, remain invaluable as FISH and other related technologies are used to complement them. The question of resolution of subtle translocation by SKY or M-FISH is an important issue. In one of our cases with a complex karyotype, rearrangements involving chromosomes 2,8 , and 9 were identified, but insertional translocation of c-myc from $8 \mathrm{q} 24.1$ to 3 p 21 could not be identified by SKY. ${ }^{14}$ It was concluded that insertions of this type can best be detected by locus specific probes in conjunction with high resolution $G$ banding. The resolution of SKY (and M-FISH) for insertional translocation appears similar to whole chromosome painting probes, approximately 1 megabase.

M-FISH was introduced by Speicher et al. ${ }^{1}$ based on the analysis of normals and a series of abnormal cases. M-FISH has not been used for routine clinical practice since the software and reagents have become available just recently. We have set up M-FISH by using the same method of processing that we have used for whole chromosome painting probes for a number of years. M-FISH software is now available from several commercial companies. The karyotype capture and analysis time ranged from 10 to 20 minutes (which is at least double of painting probes) and we had no problem in processing specimens just as we do for whole chromosome painting probes from any tissue type. At the present time the cost of a commercially available M-FISH probe set is significantly higher than chromosome paint probes.

In Cases 2, 3, 4, and 5, the most rewarding experience of $M$ FISH analysis (as it is with SKY) was the ability to rapidly 
identify the markers or the unidentified segments of the derivative chromosomes. In each of these cases, the abnormalities were confirmed by whole chromosome painting probes or locus specific probes. M-FISH was used to analyze a complex karyotype from the bone marrow of a hematologic disorder patient. In this case, multiple markers and derivative chromosomes, including one that originated from three chromosomes, were identified. Sequential GTG and individual paint probes, used one or two at a time, instead of M-FISH or SKY would be cumbersome and very time consuming to resolve such markers or derivative chromosomes and complex karyotypes.

The M-FISH has similar limitations as SKY. The insertional translocation of $\mathrm{c}-\mathrm{myc}$ from $8 \mathrm{q} 24.1$ to $3 \mathrm{p} 21$ could not be detected as indicated earlier by SKY. We could not detect it by $\mathrm{M}$-FISH either (unpublished information). Both in SKY and $\mathrm{M}$-FISH, the acrocentric short arms and highly repetitive DNA sequences of constitutive heterochromatins remain unlabeled. However, the acrocentric fluorophores often cross hybridize to the acrocentric short arms in M-FISH (Figures 1-4). Additional technical problems are the "graying out" at break or fusion points due to overlapping of fluorophore combinations of different chromosomes. Some rare chromosomal combinations can also generate interpretation problems. For instance, at breakpoint junctions of chromosomes 3 (aqua) and 5 (gold and far red) the combined fluorophores are identical to chromosome 10 (aqua, gold, and far red). This can cause an interpretive challenge. These problems can be resolved, however, by use of individual chromosome paint probes. The future improvements of the reagents and software can address some of these problems more effectively.

We routinely process G-banded spreads sequentially for painting probes. ${ }^{15,16}$ However, we have experienced difficulty in M-FISH analysis of previously G-banded cells (Figure 3) in an earlier version of $\mathrm{M}$-FISH reagents. Other technical problems were encountered: The hybridization for M-FISH needs to be optimal, because distinction of specific fluorophore signature is difficult when it is suboptimal. Some classification colors are difficult to discriminate, especially when subtle detection is desired. For instance, the classification color of chromosome 5 (involved in 3-way translocation) was changed to white (Figure 4) for a more distinct detection involving the translocation to chromosome 17.
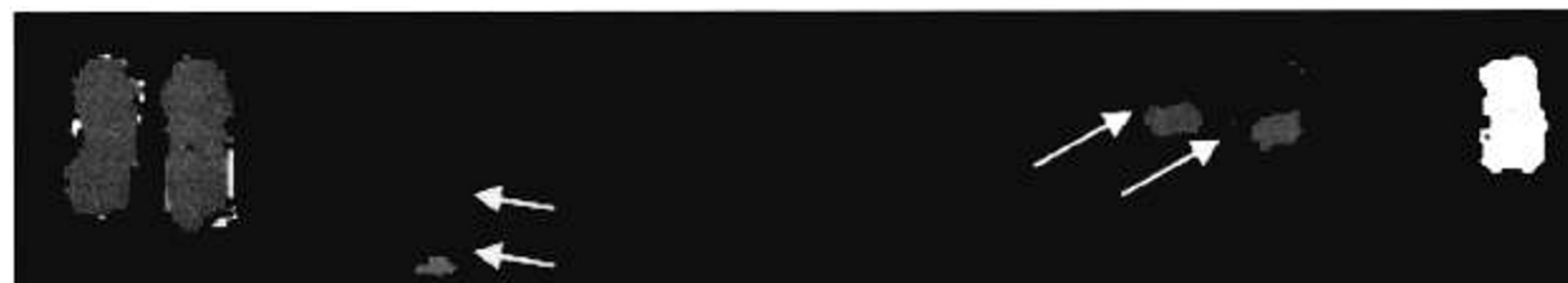

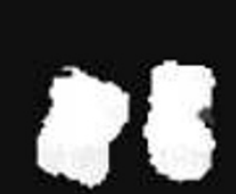

7
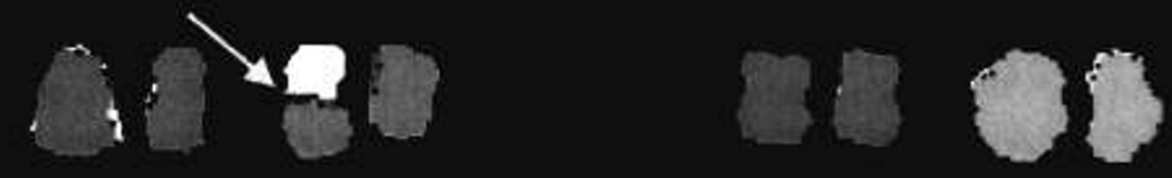

1.

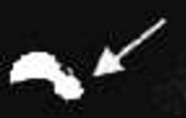

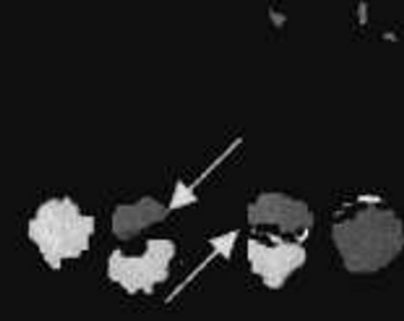

14

Fig. 4 A complex karyotype from the bone marrow analysis of an 85-ycar-old was: 44-46, XX, add(2)(q37), $-4,-5$, add $(9)(\mathrm{p} 11), \mathrm{add}(17)(\mathrm{p} 11.2)$, add $(19)(\mathrm{p} 13.1),-20,-22,+2$. $4, \mathrm{mar}[\mathrm{cp} 19] / 46, \mathrm{XX}(1)$. M-FISH identified add(2) to be $t(2 ; 4 ; 9)$ two markers to be $t(4: 22) \times 2$, add $(4)$ was $t(5 ; 9)$, add $(17)$ was $t(5 ; 17)$, add 191 was $t(19 ; 2(1)\}$ and another morker was $t(19 ; 20)$ that was undetectatie by banded chromosome anulysis. 
We have used $\mathrm{M}$-FISH with relative ease and efficiency in a variety of tissue types. $M$-FISH requires special filter sets, computer software, and reagents. The image capture and analysis is significantly more labor intensive compared with chromosome specific paint probes. However, there really is no efficient option but to scan all 24 chromosomes simultaneously by SKY or M-FISH for markers, derivative chromosomes, or complex karyotypes that cannot be resolved by high resolution banding. In many instances, individual chromosome painting probes or locus specific FISH probes are necessary to confirm or further delineate the results.

\section{References}

1. Speicher MR, Ballard SG, Ward DC. Karyotyping human chromosomes by combinatorial multi-fluor FISH. Nat Genet 1996:12:368-375

2. Eils R, Uhrig S, Saracoglu K, Satzler K', Bolzer A, Peterson I, Chassery IM, Gancer M. Speicher MR. An optimized, fully automated system for fast and accurate identification of thromosome rearrangements by multiplex-FISH (M-FISH). Cytogenet Cell Genet 1998:82:160-171.

3. Sihrock E, duManoir S, Veldman T, Schoell B, Wienberg J. Ferguson-Smith MA, Ning Y, Ledbetter DH, Bay-Am I, Soenksen D, Garini Y, Ried T. Multicolor spectral karyotyping of human chromosomes. Science 1996:273:494-497.

4. Spurbeck IL, Zinsmeister AR, Meyer KJ, Jalal SMI. Dynamics of chromosome spreading. Am / Med Genet 1996;61:387-393.

5. Warburton D. De novo structural rearrangements: implications for prenatal diagnosis. Wiley AM, Carter IP, Kelly S, Porter IH, editors. Problems in diagnosis and counseling. New York: Academic Press, 1982;63-75.
6. Warburton D. De novo balanced chromosome rearrangements and extra marker chromosomes. Identified at prenatal diagnosis: Clinical significance and distribution of breakpoints. Am I Hum Genet 1991:49:995-1013.

7. Brondum-Nielsen K, Mikkelsen M. A 10-year survey, 1980-1990, of prenatally diagnosed small supernumerary marker chromosomes, identified by FISH analysis. Outcome and follow-up of 14 cases diagnosed in a series of 12,699 prenatal samples. Prenat Diagn 1995;15:615-619.

8. Callen DF, Eyre H, Yip M-Y, Frcemantle J, Haan EA. Molecular cytogenetic and clinical studies of 42 patients with marker chromosomes. Am J Med Genet 1992;43:709-715.

9. Crolla IA. FISH and molecular studies of autosomal supernumerary marker chromosomes excluding those derived from chromosome 15: II. Review of literature. Am J Meit Genet 1998;75:367-381.

10. Crolla JA, Long F, Rivera H, Dennis NR. FISH and molecular study of autosomal supernumerary marker chromosomes excluding those derived from chromosome 15 and 22: I. Results of 26 new cases. Am / Med Genet 1998;75:355-366.

11. Schwartz S, Depinet TW, Leane-Cox J, Isada NB, Karson EM, Park VM, Pasztor LM, Sheppard LC, Stallard R, Wolff DJ, Zinn AB, Zurcher VL, Zackowski JL. Sex chromosome markers: Characterization using fluorescence in situ hybridization and review of literature. Am / Med Genet 1997:71:1-7.

12. Vierbach R, Engels $\mathrm{H}$, Gamerdinger U, Hansmann M. Delineation of supernumerary marker chromosomes in 38 patients. Am J Med Genet 1998;76:351-358.

13. Reid T, Schrock E, Ning Y, Wienberg J. Chromosome painting: A useful art. Hum Mol Genet 1998:7:1619-1626.

14. Schrock E, Veldman T, Padilla-Nash H, Ning Y, Spurbeck I, Jalal S, Shaffer LG, Papenhausen P, Kozma C, Phelan MC, Kjeldsen E, Schonberg SA, O'Brian P, Biesecker L, du Manoir S, Ried T. Spectral karyotyping refines cytogenetic diagnostics of constitutional thromosomal abnormalities. Hum Genet 1997;101:255-262.

15. Jalal SM, Law M, Christensen ER, Spurbeck JL, Dewald GW. Method for sequential staining of GTL-banded metaphases with fluorescent-labeled chromosome specific paint probes. Am J Med Genet 1993;46:98-103.

16. Jalal SM, Law ME, Dewald GW. Atlas of whole chromosome paint probes: Normal patterns and utility for abnormal cases. Mayo Foundation 1996.

\section{Erratum}

The correct footnote for the Policy Statement "Duty to Recontact" which appeared in Genetics in Medicine 1999;1:171-172, should read as follows:

"The Board of Directors of the College authorized publication of this committee statement to generate discussion on important policy matters. This statement does not represent an official policy statement of the American College of Medical Genetics regarding the duty to recontact. Although the statement represents a consensus opinion of the Social Ethical and Legal Issues Committee, the agreement was not unanimous among the committee." 